\title{
Bacillus and Clostridium Spore Attachment/ Entry of Human Colon Cells
}

\author{
B. Panessa-Warren, G.T.Tortora*, B.Ghebrehiwet**, S.S.Wong+ and J.B.Warren++
}

Biology Dept., Brookhaven National Laboratory, Upton,NY 11973, *Clinical Microbiology Lab. University Hospital and School of Health Technol. \& Management, SUNY StonyBrook, NY 11794, **Dept. of Medicine, SUNY at Stony Brook, NY 11794, +Dept.of Chemistry, SUNY Stony Brook, NY 11794 \& Materials and Chemical Sciences Dept., Brookhaven National Lab., Upton,NY,11973, ++Instrumentation Division, Brookhaven National Lab., Upton, NY 11973

With the use of anthrax spores in biowarfare, it is now evident that there are information gaps in understanding bacterial spore attack of mammalian cells, in the absence of mature bacterial cells. This investigation describes the attachment-entry process of two pathogenic endospores, an anthrax model (B.cereus) and C. difficile (a known pathogen causing antibiotic induced pseudomembranous colitis as well as nosocomial diarrheas worldwide). By using carbon nanoloops functionalized with monoclonal anti-gClq-receptor protein (mAb 60.11), a known invasion protein for Listeria monocytogenes $(1,2)$, this investigation also presents data on the role that gClq-R plays for spore attachment and entry.

Recently Helgason et al. (3) demonstrated that the genome of B.anthracis is virtually identical to that of B.cereus with the exception of the their respective toxin production. Therefore we used a blood isolate of B.cereus from an IV-drug user as an anthrax model, to study the attachmententry process of this type of spore. For comparison, pathogenic spores of C. difficile ATCC43594 were similarly studied.

Monolayers of human colon cells (Caco-2 cells) grown on $6 \mathrm{~mm}$ coverslips in shell vials (Diagnostic Hybrids Inc., Athens, Ohio), were gently swirled on a rotary shaker at $36^{\circ} \mathrm{C}$ during

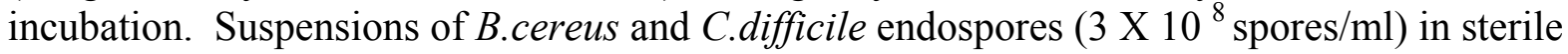
water were used to innoculate each shell vial. Spore inoculated monolayers were incubated for 1.5, 2.5 and 3.5 hours aerobically for B.cereus, and anaerobically for C.difficile. After incubation, aseptically removed media from each vial was analyzed for unattached spores, contaminants and dead detached Caco-2 cells. Carbon nanoloops $(25-35 \mathrm{~nm})$ were functionalized with $\mathrm{mAb}$ to gClq-R $(1,2,4,5)$. Monolayers were preincubated for 20 minutes with functionalized nanoloops prior to the introduction of bacterial spores. As "Controls", nanoloops functionalized with (1) purified gClq-R protein; or (2) a non-gClqR-binding mouse IgG, were used to identify the incidence of non-specific immuno-probe binding. All EM spore-cell preparations were fixed in $3 \%$ glutaraldehyde in $0.1 \mathrm{M}$ cacodylate buffer, or ruthenium red-glutaraldehyde, followed by post fixation in aqueous osmium tetroxide. TEM samples were dehydrated in acetone, embedded in Epon, thin sectioned and imaged at $80 \mathrm{KV}$ using a Philips 300. For field emission SEM, samples were fixed and dehydrated as described above, dried by the critical point method, Pt 3-4nm coated and viewed at 5 or $10 \mathrm{KV}$ using an AMRay FE1845. A Digital Instruments Nanoscope IIIa Multimode AFM was used to analyze nanoloop preparations.

When anti-gClq-R functionalized carbon nanoloops were used to pretreat cells before bacterial innoculation, no binding was observed. However functionalized-nanoloop markers attached profusely only to spore associated microvilli, suggesting that the presence of spores seems to trigger two simultaneous events: the appearance of larger more profuse microvilli; and 
the expression of $\mathrm{gClqR}$ on the spore-associated microvilli. In both C.difficile (Fig.1), and B.cereus (Fig.2) incubated with Caco-2 cells, spore outer membranes (exosporia) attached rapidly to host cell microvilli. Following 2.5-3 hrs incubation, empty exosporial membranes were found attached to surface microvilli (Fig.1\&2). Localization of gClq-R on the host cell surface, suggested exosporial membrane attachment at gClq-R expressed sites. By TEM the germinating spore protoplast which appears as an electron dense body, could be seen directly entering the surface of the host cell without phagocytosis. Although a delicate membrane was seen around the spore protoplast within the host cell cytoplasm, no bacterial cell wall had formed. These spores can exit their exosporia and spore coats, enter and pass into host cells, travel within host cell cytoplasm, while concurrently postponing the completion of germination. This spore transitional form within cytoplasm may explain how anthrax spores, inhaled in the lung, do not germinate until they are engulfed by circulating macrophages; and why C.difficile infections may require repeated courses of treatment to kill hidden spore material deep within infected colon cells.

\section{References}

[1]B.Ghebrehiwet et al.,Immunological Rev.180 (2001)65.

[2]L.Braun et al.EMBO,19 (2000) 1458.

[3]E.Helgason et al.,Appl.Rev.Microbiol.66(2000) 2627.

[4]B.Panessa-Warren et al.,Microscopy \& Microanalysis 2002, Springer Verlag (NY)(2002).

[5]B.Panessa-Warren et al.,Proceedings of the American Chemical Society, New Orleans,Louisiana, March (2003).

[6]This work was supported by DOE grant DE-ACO2-98CH10886.

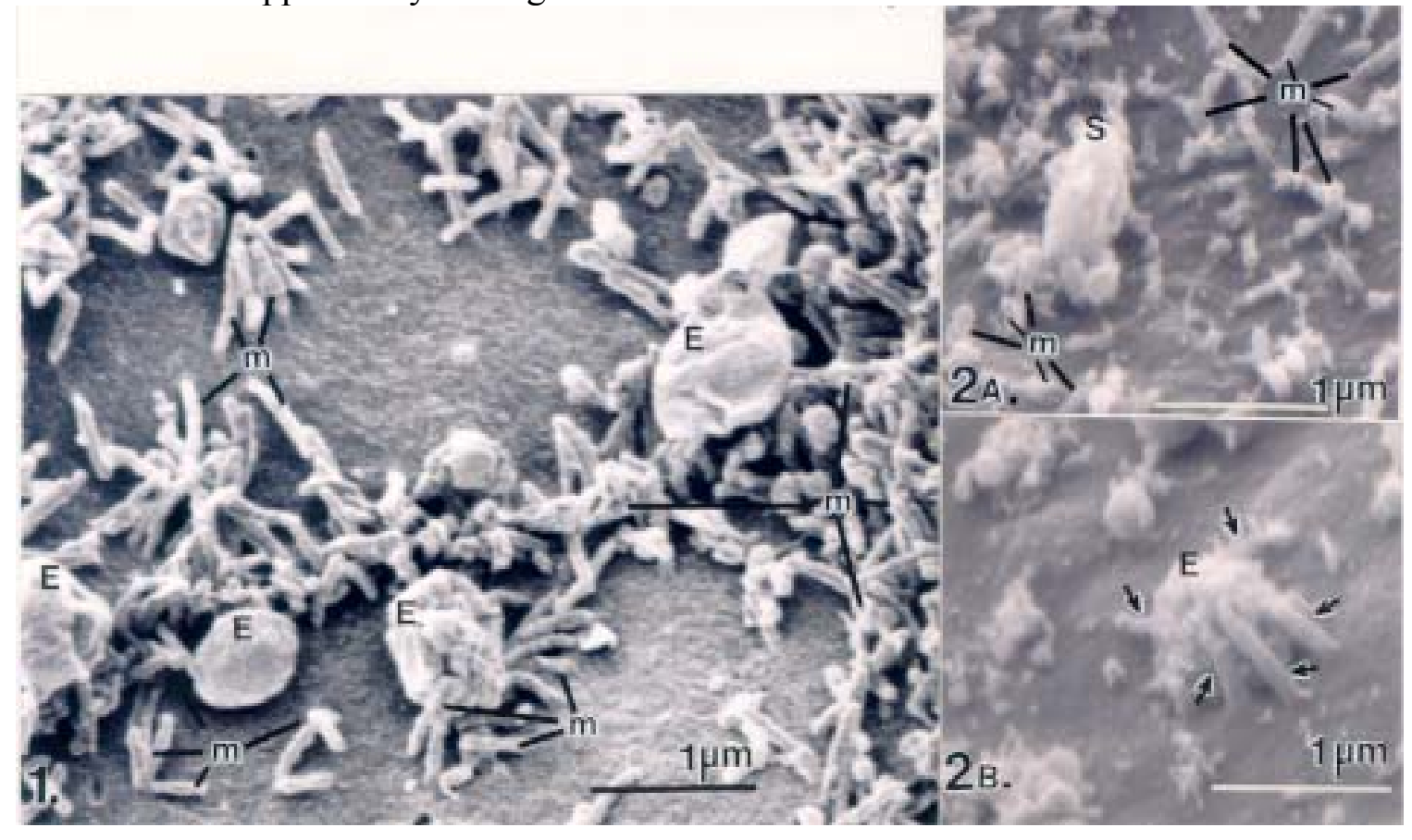

Fig.1.C.difficile empty exosporia(E) attached to Caco-2 microvilli (m) at $3 \mathrm{hr}$ incubation. Fig.2.B.cereus spores (S) newly attached to Caco-2 microvilli (2A), and (2B) empty exosporium (E) firmly attached to Caco-2 microvilli (arrows) after $3 \mathrm{hr}$ incubation. Delicate hair-like extensions on microvilli mark sites of gClq-receptor protein $(2 \mathrm{~A}, \mathrm{~B})$. 
https://doi.org/10.1017/S1431927603446898 Published online by Cambridge University Press 